\title{
Sidelobe Suppression for Robust Beamformer Via the Mixed Norm Constraint
}

\author{
Yipeng Liu • Qun Wan
}

Published online: 6 April 2011

(C) Springer Science+Business Media, LLC. 2011

\begin{abstract}
Applying a sparse constraint on the beam pattern has been suggested to suppress the sidelobe of the minimum variance distortionless response (MVDR) beamformer recently. To further improve the performance, we add a mixed norm constraint on the beam pattern. It matches the beam pattern better and encourages dense distribution in mainlobe and sparse distribution in sidelobe. The obtained beamformer has a lower sidelobe level and deeper nulls for interference avoidance than the standard sparse constraint based beamformer. Simulation demonstrates that the SINR gain is considerable for its lower sidelobe level and deeper nulling for interference, while the robustness against the mismatch between the steering angle and the direction of arrival (DOA) of the desired signal, caused by imperfect estimation of DOA, is maintained too.
\end{abstract}

Keywords Robust beamforming · Sidelobe suppression · Mixed norm constraint · Sparse constraint

\section{Introduction}

Multiple-antenna systems have received a lot of attention from both the wireless industry and academia, because of their strong potential in realizing high date rate wireless communications in next generation wireless networks. A beamformer is a versatile form of spatial filtering. It uses multiple antenna systems to separate signals that have overlapping frequency spectra but originate from different spatial locations. Beamforming has become a key technique in current and future wireless communications [1].

Y. Liu $(\varangle) \cdot$ Q. Wan

Electronic Engineering Department, University of Electronic Science and Technology of China, Chengdu 611731, China

e-mail: liuyipeng@uestc.edu.cn

Q. Wan

e-mail: wanqun@uestc.edu.cn 
The minimum variance distortionless response (MVDR) beamformer has been considered as a popular method for enhancing the signal from the desired direction while suppressing all signals from other directions as well as the background noise [1], but its relatively high sidelobe level would lead to significant performance degradation, especially with the unexpected increase in interference or background noise [2]. To enhance the robustness in the presence of array steering vector errors, doubly constrained robust capon beamformer used a norm constraint on the weight vector to improve the robustness [3]. To achieve a faster convergence speed and a higher steady state signal to interference plus noise ratio (SINR) [4] constrains its weight vector to a specific conjugate symmetric form. In [5], fully complex-valued radial basis function (RBF) network with the fully complex-valued activation function is used in MVDR beamformer to short the convergence period. However, high sidelobe is another drawback, which would result in deep degradations in the case of unexpected interferences or an increase in noise power [2]. In order to provide sidelobe suppression and angle mistmatch robustness for an MVDR beamformer, a sparse constraint on the whole beam pattern was recently proposed in [6], and a robust beamformer with the sidelobe suppressed was obtained.

In [6], the sparse constraint was added equally on both the mainlobe and the sidelobe. But the expected beam pattern would enjoy most of the high array gains in the mainlobe, and the array gains in the mainlobe are in dense distribution. The constraint should encourage dense distribution in mainlobe and sparse distribution in sidelobe. To further enhance the performance, a mixed norm constraint is incorporated to match the expected beam pattern better. Numerical evaluations show that the proposed beamformer achieves a lower sidelobe level, deeper nulls for interference avoidance.

\section{MVDR Beamformer}

The signal received by a uniform linear array (ULA) with $M$ antennas can be represented by an $M$-by-1 vector, $\mathbf{x}(k)$, the expression of which is given by

$$
\mathbf{x}(k)=s(k) \mathbf{a}\left(\theta_{0}\right)+\sum_{j=1}^{J} \beta_{j}(k) \mathbf{a}\left(\theta_{j}\right)+\mathbf{n}(k)
$$

where $k$ is the index of time, $J$ is the number of interference sources, $\mathrm{s}(k)$ and $\beta_{i}(k)$ (for $j=1, \ldots, J)$ are the amplitudes of the SOI (signal of interest) and interfering signals at time instant $k$, respectively, $\theta_{l}$ (for $l=0,1, \ldots, J$ ) are the DOAs of the SOI and interfering signals, $\varphi_{l}=(2 \pi d / \lambda) \sin \theta_{l}$, with $d$ being the distance between two adjacent antennas and $\lambda$ being the operating wavelength [1], i.e., the wavelength of the SOI, and $\mathbf{n}(k)$ is the additive white Gaussian noise (AWGN) vector at time instant $k ; \mathbf{a}(\theta)$ is the steering vector in the angle $\theta$, with its $m$-th element $\exp \left(j(m-1) \frac{2 \pi d}{\lambda} \sin \theta\right)$.

The output of a beamformer for the time instant $k$ is then given by

$$
y(k)=\mathbf{w}^{H} \mathbf{x}(k)=s(k) \mathbf{w}^{H} \mathbf{a}\left(\theta_{0}\right)+\sum_{j=1}^{J} \beta_{j}(k) \mathbf{w}^{H} \mathbf{a}\left(\theta_{j}\right)+\mathbf{w}^{H} \mathbf{n}(k)
$$

where $\mathbf{w}$ is the $M$-by-1 complex-valued weighting vector of the beamformer.

The MVDR beamformer is designed to minimize the total array output energy, subject to a linear distortionless constraint on the SOI. The weighting vector of the MVDR beamformer [1] is given by 


$$
\mathbf{w}_{\text {MVDR }}=\underset{\mathbf{w}}{\arg \min }\left(\mathbf{w}^{H} \mathbf{R}_{x} \mathbf{w}\right), \text { s.t. } \mathbf{w}^{H} \mathbf{a}\left(\theta_{0}\right)=1
$$

where $\mathbf{R}_{x}$ is the $M$-by- $M$ covariance matrix of the received signal vector $\mathbf{x}(k)$, and $\mathbf{w}^{H} \mathbf{a}\left(\theta_{0}\right)=1$ is the distortionless constraint applied on the SOI.

\section{The Mixed Norm Constraint Beamformer}

In order to suppress the sidelobe level of the conventional MVDR beamformer, a sparse constraint on the whole beam pattern was suggested in [6]. Accordingly, the weighting vector of the improved MVDR beamformer based on a sparse constraint (SC) is given by

$$
\mathbf{w}_{\mathrm{SC}}=\underset{\mathbf{w}}{\arg \min }\left(\mathbf{w}^{H} \mathbf{R}_{x} \mathbf{w}+\gamma_{1}\left\|\mathbf{w}^{H} \mathbf{A}\right\|_{p}^{p}\right) \text {, s.t. } \mathbf{w}^{H} \mathbf{a}\left(\theta_{0}\right)=1
$$

where $\gamma_{1}$ is the factor that controls the tradeoff between the minimum variance constraint on the total array output energy and the sparse constraint on the beam pattern, The $M$-by- $N$ matrix $\mathbf{A}$ is the steering matrix with $\alpha_{n} \mathrm{~s}(n=1,2, \ldots, N)$ being the sampled angles in the $\left[-90^{\circ}, 90^{\circ}\right]$, and it covers all the $N$ steering vectors for all possible interference with DOA in the sampling range, with $\alpha_{0}$ being the DOA of the SOI as defined in (1), i.e.

$$
\begin{gathered}
\mathbf{A}=\left[\begin{array}{ccc}
1 & \cdots & 1 \\
\exp \left(j \varphi_{1}\right) & \cdots & \exp \left(j \varphi_{N}\right) \\
\vdots & \ddots & \vdots \\
\exp \left(j(M-1) \varphi_{1}\right) & \cdots & \exp \left(j(M-1) \varphi_{N}\right)
\end{array}\right] \\
\varphi_{n}=\frac{2 \pi d}{\lambda} \sin \alpha_{n}, \text { for } n=1, \cdots, N
\end{gathered}
$$

and $\|\mathbf{x}\|_{p}=\left(\sum_{i}\left|x_{i}\right|^{p}\right)^{1 / p}$ is is the $\mathscr{C}_{p}$ norm of a vector $\mathbf{x}$. When $0 \leq p \leq 1$, the $\mathscr{C}_{p}$ norm provides a measurement of sparsity for $\mathbf{x}$. The smaller the value of $\|\mathbf{x}\|_{p}^{p}$ is. the sparser the vector $\mathbf{x}$ is, It means that the number of trivial entries in $\mathbf{x}$ is larger [6]. When $p=1,(4)$ is an second order cone programming (SOCP), and can be solved efficiently.

The optimal weighting vector indicated by (4) can be found by an adaptive iteration algorithm [6,7]. When $p=1$, a simpler way called basis pursuit [8], can solve (4) efficiently.

In (4), the sparse constraint operates for all the array gains $\mathbf{w}^{H} \mathbf{A}$ in all the possible values of DOA from -90 to $90^{\circ}$, i.e. it enforces sparse distribution of the array gains in both the mainlobe and the sidelobe. However, the array gains are not in standard sparse distribution, but in dense distribution in the mainlobe and in sparse distribution in the sidelobe. Here instead of the standard sparse constraint, an mixed norm constraint with different norms on different lobes can be added to the MVDR beamformer to improve the performance. It can be formulated as

$$
\begin{gathered}
\mathbf{w}_{\mathrm{MNB}}=\underset{\mathbf{w}}{\arg \min }\left[\mathbf{w}^{H} \mathbf{R}_{x} \mathbf{w}+\gamma_{2}\left(\left\|\mathbf{w}^{H} \mathbf{A}_{M}\right\|_{\infty}+\left\|\mathbf{w}^{H} \mathbf{A}_{S}\right\|_{1}\right)\right] \\
\text { s.t. }{ }^{H} \mathbf{a}\left(\theta_{0}\right)=1
\end{gathered}
$$

where

$$
\begin{aligned}
& \mathbf{A}_{M}=\left[\mathbf{a}\left(\theta_{-b}\right) \cdots \mathbf{a}\left(\theta_{0}\right) \cdots \mathbf{a}\left(\theta_{+b}\right)\right] \\
& \mathbf{A}_{S}=\left[\mathbf{a}\left(\theta_{-90}\right) \cdots \mathbf{a}\left(\theta_{-b-1}\right) \mathbf{a}\left(\theta_{+b+1}\right) \cdots \mathbf{a}\left(\theta_{+90}\right)\right]
\end{aligned}
$$


$\mathbf{A}_{M}$ and $\mathbf{A}_{S}$ are sub-matrices of the steering matrix $\mathbf{A} . \mathbf{A}_{M}$ is composed of $2 b+1$ steering vectors with the sampled angles in the mainlobe; while $\mathbf{A}_{S}$ is constituted with the rest of the steering vectors in $\mathbf{A}$. The product $\mathbf{w}^{H} \mathbf{A}_{M}$ indicates array gains of the mainlobe in the beam pattern, and $\mathbf{w}^{H} \mathbf{A}_{S}$ indicates array gains of the sidelobe. The width of the first block is $2 b+1$ corresponding to the mainlobe; and the other block's width is corresponding to the sidelobe. $\gamma_{2}$ is the weighting factor that controls the tradeoff between the minimum variance constraint on the total array output energy and the mixed norm constraint on the beam pattern to shape the beam pattern, $b$ is an integer representing the bounds of the mainlobe block. The minimization of $\left\|\mathbf{w}^{H} \mathbf{A}_{M}\right\|_{\infty}+\left\|\mathbf{w}^{H} \mathbf{A}_{S}\right\|_{1}$ is the mixed norm constraint.

As the mixed norm constraint are used in (7), we name it as the mixed norm beamformer (MNB). To make a difference, the beamformer (4) which was proposed in [6] is named as the standard sparse beamformer. Since the objective function of MNB is convex, the optimal $\mathbf{w}_{\text {MNB }}$ can be solved out by cvx [9] and SeDuMi [10].

In the mixed norm beamformer (7), the term $\left\|\mathbf{w}^{H} \mathbf{A}_{S}\right\|_{1}$ enforces the sparse distribution of the array gains in the sidelobe. That is to say, the number of the non-trivial array gains is much less than trivial gains'. And the term $\left\|\mathbf{w}^{H} \mathbf{A}_{M}\right\|_{\infty}$ lets the array gains in the mainlobe be dense. And that is to say, the amplitude difference of the array gains in this area is little. Then the obtained beam pattern would be like this: most of the non-trivial elements (array gains) are in the mainlobe; and the rest trivial elements are in the sidelobe. Thus, with most of entries in the mainlobe being non-trivial, the angle mismatch would not seriously degenerate the performance; and with most of entries in the sidelobe being trivial, the effect of interferences and background noise would decrease.

To illustrate the why the $\ell_{1}$ norm encourage sparse distribution and the $\ell_{\infty}$ norm encourage dense distribution, a simple geometry is given in Fig. 1. In the figure the minimization of the $\ell_{1}$ norm of a two dimensional vector, the $\ell_{2}$ norm of a two dimensional vector and the $\ell_{\infty}$ norm of a two dimensional vector are represented as a smallest rhombus, a smallest circular and a smallest exact square, respectively. The distortionless constraint can be represented as a line. The optimal solution for the minimum norm $\left\|\mathbf{w}^{H} \mathbf{A}\right\|_{p},(p=1,2,+\infty)$ subject to the distortionless constraint $\mathbf{w}^{H} \mathbf{a}\left(\theta_{0}\right)=1$ would be the tangent point where the line and the

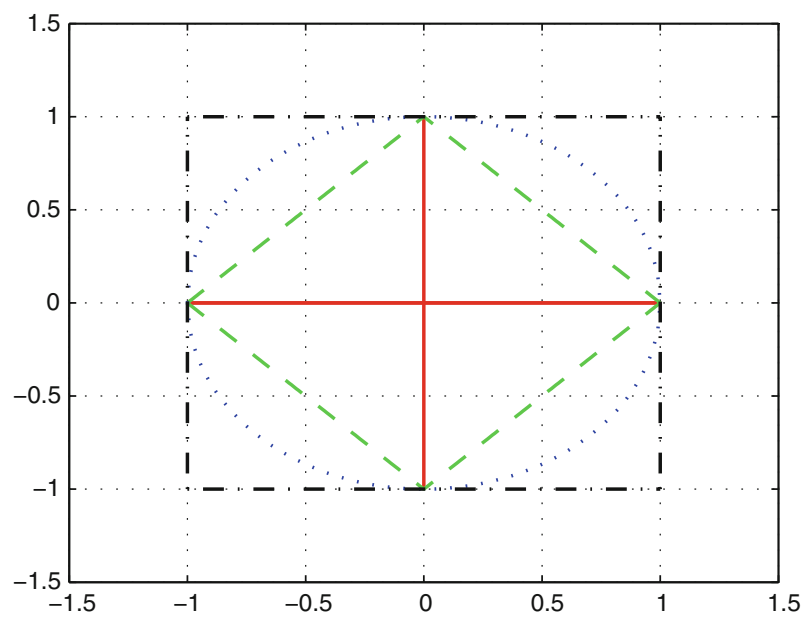

Fig. 1 The geometry demonstration of the $\ell_{1}$ norm, $\ell_{2}$ norm and $\ell_{\infty}$ norm in the place (two-dimensional space) 
curve (rhombus, circular, square) meet. Therefore, for the $\ell_{1}$ norm minimization situation, two entries in the solution would has very different absolute values with high probability; and for the $\ell_{\infty}$ norm minimization situation, two entries in the solution would has considerable similar absolute values with high probability.

The proposed MNB (7) fits the beam pattern better than the standard sparse beamformer in practice, and the performance of the proposed MNB would be improved.

\section{Simulation Results}

In the simulations, a ULA with 8 half-wavelength spaced antennas is considered. The AWGN at each sensor is assumed spatially uncorrelated. The DOA of the SOI is set to be $0^{\circ}$, and the DOAs of three interfering signals are set to be $-30,30$, and $70^{\circ}$, respectively. The signal to noise ratio (SNR) is set to be $10 \mathrm{~dB}$, and the interference to noise ratios (INRs) are assumed to be 20,20 , and $40 \mathrm{~dB}$, in $-30,30$, and $70^{\circ}$, respectively. 100 snapshots are used for each simulation. Without loss of generality, $p$ is set to be $1 ; b$ is set to be 23; and $\gamma_{1}, \gamma_{2}$ are all set to be 10 . The matrix A consists of all steering vectors in the DOA range of $\left[-90^{\circ}, 90^{\circ}\right]$ with the sampling interval of $1^{\circ}$.

To quantify the performance enhancement by the mixed norm constraint. The SINR is calculated via the following formula:

$$
\operatorname{SINR}(b)=\frac{\sigma_{s}^{2} \mathbf{w}(b)^{H} \mathbf{a}\left(\theta_{0}\right) \mathbf{a}^{H}\left(\theta_{0}\right) \mathbf{w}(b)}{\mathbf{w}(b)^{H}\left(\sum_{j=1}^{J} \sigma_{j}^{2} \mathbf{a}\left(\theta_{j}\right) \mathbf{a}^{H}\left(\theta_{j}\right)+\mathbf{Q}\right) \mathbf{w}(b)}
$$

where $\sigma_{s}$ and $\sigma_{j}$ are the variances of the SOI and $j$-th interference, $\mathbf{Q}$ is a diagonal matrix with the diagonal elements being the noise's variances. For a fixed $b, \mathbf{w}_{I B S B}(b)$ can be obtained via (7). Then SINR(b) can be obtained via (10). Simulations show that that from $b=1$ to $b=35$, The value of SINR increases gradually at the beginning, and achieves the maximum SINR at $b_{\text {opt }}=23$. Then it drops afterwards. We can see that from $b=0$ to $b=b_{\text {opt }}$, the SINR increases gradually and when $b>b_{\text {opt }}$, the SINR increases gradually.

Figure 2 shows beam patterns of the MVDR beamformer (3), the standard sparse beamformer (4), and the MNB (7) of 1000 Monte Carlo simulations. It is obvious that the best sidelobe suppression performance is achieved by the MNB (7). Among the three beamformers, the MNB (7) has the lowest array gain level in sidelobe area, and provides the deepest nulls in the directions of interference, i.e., $-30,30$ and $70^{\circ}$. The average received SINR by the MVDR beamformer (3), the standard sparse beamformer (4) and the MNB (7) are 1.2464, 4.6289 and $5.8712 \mathrm{~dB}$.

Figure 3 shows beam patterns of the beamformers that we have discussed, with each beamformer having a $4^{\circ}$ mismatch between the steering angle and the DOA of the SOI [11]. We can see that the MVDR beamformer has a deep notch in $4^{\circ}$, which is the DOA of the SOI. It can be explained by using the fact that the MVDR beamformer is designed to minimize the total array output energy subject to a distortionless constraint in the DOA of the SOI, so when the steering angle is in $4^{\circ}$, instead of $0^{\circ}$, the MVDR beam pattern maintains distortionless in $0^{\circ}$ while resulting in a deep null in $4^{\circ}$. This observation shows the high sensitivity of the MVDR beamformer to steering angle mismatch. Comparing beam patterns of beamformers defined in (4) and (7), we can see that the MNB (7) further suppresses sidelobe levels and deepens the nulls for interference avoidance, and has almost the same robustness against mismatch. In the case of $4^{\circ}$ mismatch, the average received SINR by the MVDR beamformer 


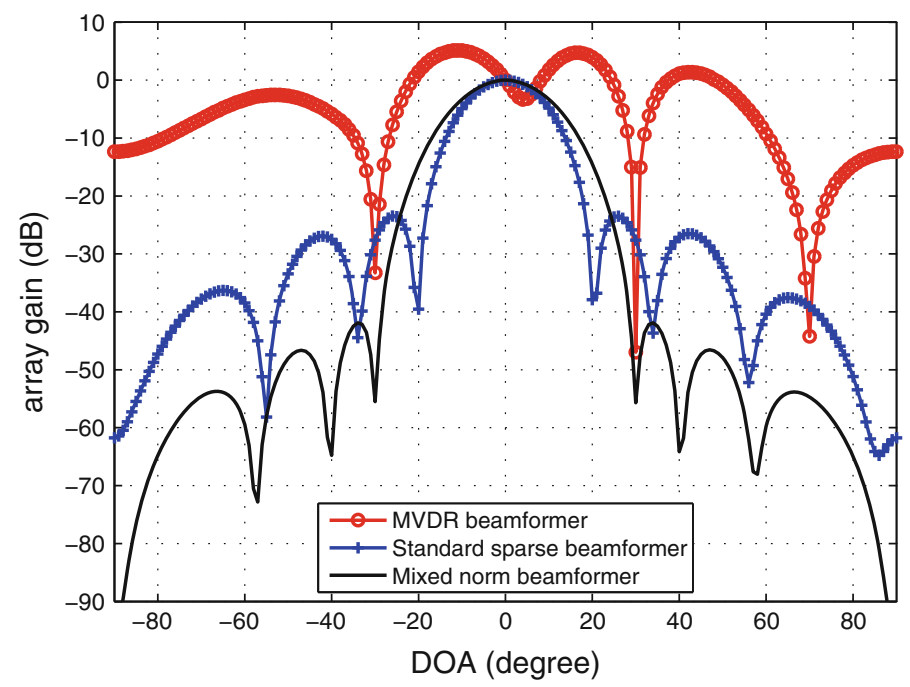

Fig. 2 Normalized beam patterns of the MVDR beamformer, standard sparse beamformer and the mixed norm beamformer, without mismatch between the steering angle and the DOA of the SOI

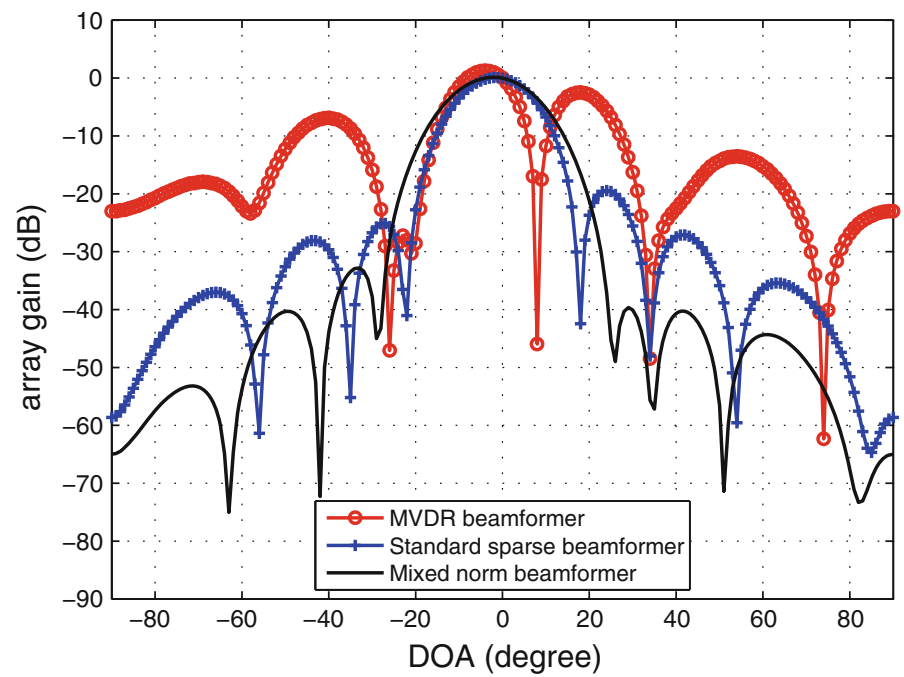

Fig. 3 Normalized beam patterns of the MVDR beamformer, standard sparse beamformer and the mixed norm beamformer, with four mismatch between the steering angle and the DOA of the SOI

(3), standard sparse beamformer (4) and the MNB (7) are $0.0005,2.0163$ and $3.2015 \mathrm{~dB}$, respectively.

When $b=0$, the MNB (7) changes into the standard sparse beamformer (4). For $b \neq 0$, the beam shaping property of the beam pattern is exploited. Our simulations also shows that the optimal block width $b$ is between 21 to 27 , when the directions of interferences are in $\left[-90^{\circ},-15^{\circ}\right)$ and $\left[15^{\circ}, 90^{\circ}\right)$. 
Thus, our proposed beamformer provides improvements in terms of sidelobe suppression, nulling for interference avoidance, while maintaining the robustness against the DOA estimation errors, with respect to existing beamformers.

\section{Conclusion}

The proposed MNB shows superiority to the MVDR beamformer and the standard sparse beamformer. It outperforms in terms of sidelobe suppression, nulling for interference avoidance, while maintaining the robustness against DOA mismatch.

Acknowledgments The authors would like to thank the anonymous reviewers. This work was supported in part by the National Natural Science Foundation of China under grant 60772146, the National High Technology Research and Development Program of China (863 Program) under grant 2008AA12Z306 and in part by Science Foundation of Ministry of Education of China under grant 109139.

\section{References}

1. Li, J., \& Stoica, P. (2006). Robust adaptive beamforming. New York: Wiley.

2. Wax, M., \& Anu, Y. (1996). Performance analysis of the minimum variance beamformer. IEEE Transactions on Signal Processing, 44(4), 928-937.

3. Li, J., Stoica, P., \& Wang, Z. (2004). Doubly constrained robust capon beamformer. IEEE Transactions on Signal Processing, 52, 2407-2423.

4. Zhang, L., Liu, W., \& Langley, R. J. (2009). A minimum variance beamformer with linear and quadratic constraints based on uniform linear antenna arrays. In Antennas and propagation conference 2009 (LAPC 2009), Loughborough,November 16-17, 2009 (pp. 585-588).

5. Savitha, R., Vigneswaran, S., Suresh, S., \& Sundararajan, N. (2009). Adaptive beamforming using complex-valued radial basis function neural networks. In 2009 IEEE region 10 conference (TENCON 2009), Singapore, January 23-26, 2009 (pp. 1-6).

6. Zhang, Y., Ng, B. P., \& Wan, Q. (2008). Sidelobe suppression for adaptive beamforming with sparse constraint on beam pattern. Electronics Letters, 44(10), 615-616.

7. Rao, B. D., Engan, K., Cotter, S. F., Palmer, J., \& Delgado, K. K. (2003). Subset selection in noise based on diversity measure minimization. IEEE Transactions on Signal Processing, 51(3), 760-770.

8. Chen, S., \& Donoho, D. (1994). Basis pursuit. 28th Asilomar Conference on Signals, Systems and Computers, 1, 41-44.

9. Grant, M., Boyd, S., \& Ye, Y. cvx user' guide for cvx version 1.1, [online] accessible at: http://www. stanford.edu/ boyd/index.html.

10. Sturm, J. (1999). Using sedumi 1.02, a matlab toolbox for optimization over symmetric cones. Optimization Methods and Software, 11(12), 625-653.

11. Chen, C., \& Vaidyanathan, P. P. (2007). Quadratically constrained beamforming robust against direction-of-arrival mismatch. IEEE Transactions on Signal Processing, 55(8), 4139-4150.

12. Stojnic, M., Parvaresh, F., \& Hassibi, B. (2009). On the reconstruction of block-sparse signals with an optimal number of measurements. IEEE Transaction on Signal Processing, 57(8), 3075-3085. 


\section{Author Biographies}
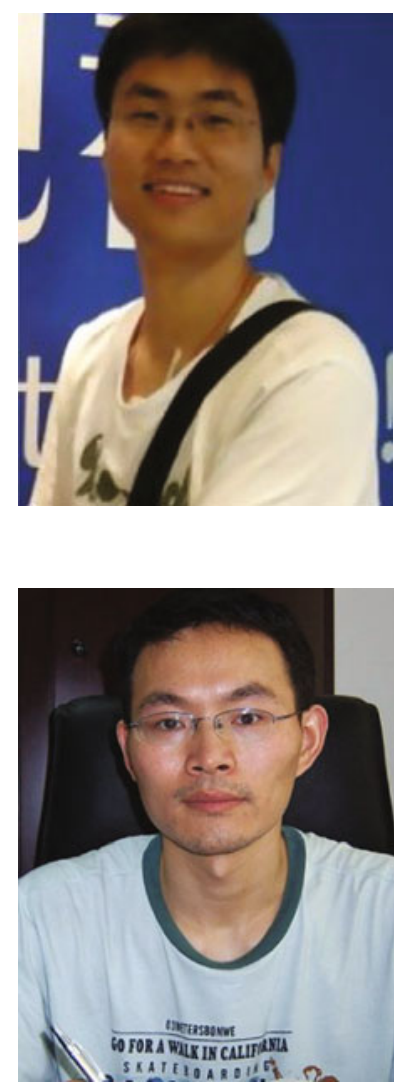

Yipeng Liu received the B.Eng. degree from University of Electronic Science and Technology of China (UESTC), Chengdu, China, in 2006. Since 2006, he has been a postgraduate with the Electronic Engineering (EE) department, UESTC, Chengdu, China. Since October 2010, he has been a visiting Ph.D. candidate at Tsinghua University (THU). His research interest focuses on compressive sensing, conformal array signal processing, wideband spectrum sensing for cognitive radio.

Qun Wan received the B.S. degree from Nanjing University in 1993, the M.S. degree from UESTC in 1996, and the Ph.D. degree from UESTC in 2001. During 2001-2002, he was a post-doctor at Tsinghua University, where he participated in cellular localization program. In 2003, he was a Technical Staff at UTstarcom. Since 2004, he has been a Professor in the Department of Electronic Engineering at the University of Electronic Science and Technology of China (UESTC). His research interests include sparse and array signal processing, mobile and indoor localization. He is a Senior Member of CIE. 